\title{
Radio Observations of Supernovae
}

\author{
Nino Panagia ${ }^{1 *}$, Kurt W. Weiler ${ }^{\dagger}$, Schuyler D. Van Dyk ${ }^{* *}$, Richard A. \\ Sramek ${ }^{\ddagger}$ and Christopher J. Stockdale ${ }^{\S}$ \\ ${ }^{*}$ STSCI, 3700 San Martin Dr., Baltimore, MD 21218, USA; panagia@stsci .edu \\ ${ }^{\dagger}$ Naval Research Laboratory, Code 7210, Washington, DC 20375-5320, USA, \\ Kurt.Weiler@nrl.navy.mil \\ ${ }^{* *}$ Spitzer Science Center/Caltech, MS 100-22, Pasadena, CA 91125, USA; \\ vandyk@ipac.caltech.edu \\ NRAO/VLA, PO Box 0, Socorro, NM 87801 USA; dsramek@nrao.edu \\ $\$$ Marquette University, Physics Dept., PO Box 1881, Milwaukee, WI 53201-1881, USA; \\ Christopher.Stockdale@marquette.edu
}

\begin{abstract}
.
Study of radio supernovae over the past 25 years includes two dozen detected objects and more than 100 upper limits. From this work it is possible to identify classes of radio properties, demonstrate conformance to and deviations from existing models, estimate the density and structure of the circumstellar material and, by inference, the evolution of the presupernova stellar wind. It is also possible to detect ionized hydrogen along the line of sight, to demonstrate binary properties of the stellar system, to detect clumpiness of the circumstellar material, and to put useful constraints on the progenitors of undetected Type Ia supernovae.
\end{abstract}

Keywords: Supernovae: General, Radio Continuum: Stars, Circumstellar Matter, Stars: Evolution, Stars: Mass Loss, Binaries: General, Supernovae: Progenitors

PACS: $98.70 . \mathrm{Dk}, 97.60 . \mathrm{Bw}, 97.60 .-\mathrm{s}, 97.80 .-\mathrm{d}$

\section{RADIO SUPERNOVAE}

A series of papers on radio supernovae (RSNe) has established the radio detection and, in a number of cases, radio evolution for approximately two dozen type $\mathrm{Ib} / \mathrm{c}$ supernovae (SNe) (Because the differences between the SN optical classes are slight - type Ib show strong He I absorption while type Ic show weak He I absorption - and there are no obvious radio differences, we shall often refer to the classes as type $\mathrm{Ib} / \mathrm{c}$.), and the rest type II SNe. A much larger list of more than 100 additional SNe have low radio upper limits (See http://rsd-www.nrl.navy.mil/7213/weiler/kwdata/rsnhead.html).

In this extensive study of the radio emission from $\mathrm{SNe}$, several effects have been noted:

- type Ia SNe are not radio emitters to the detection limit of the VLA ${ }^{2}$

- type $\mathrm{Ib} / \mathrm{c} \mathrm{SNe}$ are radio luminous with steep spectral indices (generally $\alpha<-1$;

${ }^{1}$ Also: Istituto Nazionale di Astrofisica (INAF), Via del Parco Mellini 84, I-00136, Rome, Italy; and Supernova Ltd., OYV \#131, Northsound Road, Virgin Gorda, British Virgin Islands.

2 The VLA telescope of the National Radio Astronomy Observatory is operated by Associated Universities, Inc. under a cooperative agreement with the National Science Foundation.

CP924, The Multicolored Landscape of Compact Objects and their Explosive Origins - Cefalu 2006, edited by T. Di Salvo, L. A. Antonelli, G. L. Israel, L. Piersanti, A. Tomambe, L. Burderi, F. Fiore, G. Matt, and M. T. Menna (O) 2007 American Institute of Physics 978-0-7354-0434-2/07/\$23.00 
$S \propto v^{+\alpha}$ ) and have a fast turn-on/turn-off, usually peaking at $6 \mathrm{~cm}$ near or before optical maximum.

- type II SNe show a range of radio luminosities with flatter spectral indices (generally $\alpha>-1$ ) and a relatively slow turn-on/turn-off, usually peaking at $6 \mathrm{~cm}$ significantly after optical maximum.

Measurements of the multi-frequency radio light curves and their evolution with time show the density and structure of the CSM, evidence for possible binary companions, clumpiness or filamentation in the presupernova wind, mass-loss rates and changes therein for the presupernova stellar system and, through stellar evolution models, estimates of the ZAMS presupernova stellar mass and the stages through which the star passed on its way to explosion.

\section{EMISSION MODELS}

All known RSNe appear to share common properties of:

- Nonthermal synchrotron emission with high brightness temperature.

- A decrease in absorption with time, resulting in a smooth, rapid turn-on first at shorter wavelengths and later at longer wavelengths.

- A power-law decline of the flux density with time at each wavelength after maximum flux density (optical depth $\sim 1$ ) is reached at that wavelength.

The characteristic RSN radio light curves arise from the competing effects of slowly declining non-thermal radio emission and more rapidly declining thermal or non-thermal absorption yielding a rapid turn-on and slower turn-off of the radio emission at any single frequency. This characteristic light curve shape is illustrated in Fig. 1-2 for SN 1979C and SN 1980K. Since absorption processes are greater at lower frequencies, transition from optically thick to optically thin (turn-on) occurs first at higher frequencies and later at lower frequencies.

Chevalier $[1,2]$ has proposed that the relativistic electrons and enhanced magnetic field necessary for synchrotron emission arise from the SN blastwave interacting with a relatively high density CSM which has been ionized and heated by the initial UV/Xray flash. This CSM density $(\rho)$, which decreases as an inverse power, $s$, of the radius, $r$, from the star, is presumed to have been established by a presupernova stellar wind with mass-loss rate, $\dot{M}$, and velocity, $w_{\text {wind }}$, (i.e., $\rho \propto \frac{\dot{M}}{w_{\text {wind }} r^{2}}$ ) from a massive stellar progenitor or companion. For a constant mass-loss rate and constant wind velocity $\rho \propto r^{-2}$. This ionized CSM is the source of some or all of the initial thermal gas absorption. Additionally, Chevalier [3] has proposed that synchrotron self-absorption (SSA) may play a role in some objects.

A rapid rise in the observed radio flux density results from a decrease in these absorption processes as the radio emitting region expands and the absorption processes, either internal or along the line-of-sight, decrease. Weiler et al. [26] have suggested that this CSM can be "clumpy" or "filamentary," leading to a slower radio turn-on, and Montes et al. [8] have proposed the possible presence of a distant ionized medium along the line-of-sight that is sufficiently distant from the explosion that it is unaffected by 


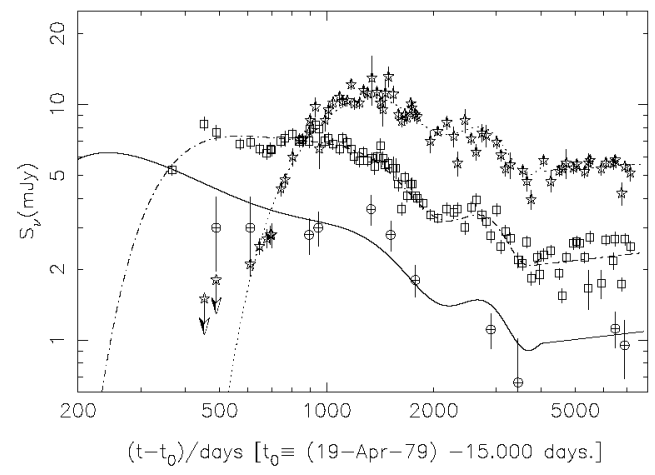

FIGURE 1. Type II SN 1979C at $2 \mathrm{~cm}$ (14.9 GHz; crossed circles, solid line), $6 \mathrm{~cm}$ (4.9 GHz; open squares, dash-dot line $)$, and $20 \mathrm{~cm}(1.5 \mathrm{GHz}$; open stars, dotted line $)$. Note that the radio flux density increases after day $\sim 4000$ and has a sinusoidal modulation before day $\sim 4000[27,28,10]$.

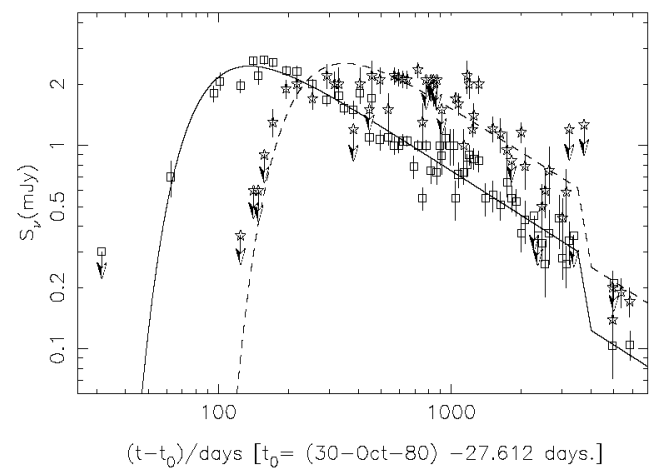

FIGURE 2. Type II SN $1980 \mathrm{~K}$ at $6 \mathrm{~cm}(4.9 \mathrm{GHz}$; open squares, solid line), and $20 \mathrm{~cm}(1.5 \mathrm{GHz}$; open stars, dashed line). Note a sharp drop in flux density after day $\sim 4000[29,9]$.

the blastwave and can cause a spectral turn-over at low radio frequencies. In addition to clumps or filaments, the CSM may be radially structured with significant density irregularities such as rings, disks, shells, or gradients.

\section{PARAMETERIZED RADIO LIGHT CURVES}

Weiler et al. [25, 26] and Montes et al. [8] adopted a parameterized model which has been updated in [31]. The model includes an intrinsic sychrotron emission, which is characterized by a spectral index $\alpha<0$ and declines with time as a power law with exponent $\beta<0\left(S_{v} \propto v^{\alpha} t^{\beta}\right)$, and an attenuation mostly produced by $f$ - $f$ absorption from the ionized CSM, but also, especially at early times, by sychrotron self-absorption. Each $i$-th absorption process is characterized by a power law decline with slope $\delta_{i}$. Various possibilities for the $f-f$ absorption are included, namely (i) homogeneous and (ii) clumpy 
or filamentary absorption by gas in the CSM, as well as (iii) the one originating in a possible foreground HII region. The homogeneous absorption is produced by an ionized medium that completely covers the emitting source, and additional attenuation may be produced by an inhomogeneous medium ("clumpy absorption"; see [11] for a more detailed discussion of attenuation in inhomogeneous media). The distant $f-f$ absorption is produced by a homogeneous medium which completely covers the source but is so far from the SN progenitor that it is not affected by the expanding SN blastwave and is constant in time.

Since it is physically realistic and may be needed in some RSNe where radio observations have been obtained at early times and high frequencies, the model also includes the possibility for an internal absorption term. This internal absorption term consists of two parts - synchrotron self-absorption, and mixed, $f-f$ absorption due to thermal gas coexisting with the non-thermal emitting electrons.

\section{RESULTS}

The success of the basic parameterization and modeling has been shown in the good correspondence between the model fits and the data for all subtypes of RSNe: e.g., type Ib SN 1983N [20], type Ic SN 1990B [22], and type II SN 1979C [27, 28, 10] and SN $1980 \mathrm{~K}([29,9]$. Note that after day $\sim 4000$, the evolution of the radio emission from both SN 1979C and SN 1980K deviates from the expected model evolution and that SN 1979C shows a sinusoidal modulation in its flux density prior to day $\sim 4000$ (se Fig. 1 and 2).

Additionally, in their study of the radio emission from SN 1986J, Weiler et al. [26] found that the simple Chevalier model could not describe the relatively slow turnon. They therefore included terms described mathematically by a clump optical depth, $\tau_{\mathrm{CSM}_{\text {clumps }}}$, in the model equations. This extension greatly improved the quality of the fit and was interpreted by [26] to represent the presence of filaments or clumps in the CSM. Such a clumpiness in the wind material was again required for modeling the radio data from SN 1988Z [23, ] and SN 1993J [24]. Since that time, evidence for filamentation in the envelopes of SNe has also been found from optical and UV observations $[5,19]$.

\section{Mass-Loss Rate from Radio Absorption}

From the Chevalier model $[1,2]$, the turn-on of the radio emission for RSNe provides a measure of the presupernova mass-loss rate to wind velocity ratio $\left(\dot{M} / w_{\text {wind }}\right)$. Weiler et al. [25] derived this ratio for the case of pure, external absorption by a homogeneous medium. However, Weiler et al. [31] propose several possible origins for absorption and generalize Eq. 16 of [25] to

$$
\frac{\dot{M}\left(\mathrm{M}_{\odot} \mathrm{yr}^{-1}\right)}{\left(w_{\text {wind }} / 10 \mathrm{~km} \mathrm{~s}^{-1}\right)}=3.0 \times 10^{-6}<\tau_{\mathrm{eff}}^{0.5}>m^{-1.5}\left(\frac{v_{\mathrm{i}}}{10^{4} \mathrm{~km} \mathrm{~s}^{-1}}\right)^{1.5}\left(\frac{t_{\mathrm{i}}}{45 \text { days }}\right)^{1.5}
$$




$$
\times\left(\frac{t}{t_{1}}\right)^{1.5 m}\left(\frac{T}{10^{4} \mathrm{~K}}\right)^{0.68}
$$

where $m$ is the exponent of the power-law growth of the SN front radius, $r \propto t^{m}$.

Since the appearance of optical lines for measuring SN ejecta velocities is often delayed a bit relative to the time of the explosion, for convenience they take the reference time to be $t_{\mathrm{i}}=45$ days. Also, because many SN measurements indicate velocities of $\sim 10,000 \mathrm{~km} \mathrm{~s}^{-1}$, one usually assumes $v_{\mathrm{i}}=v_{\text {blastwave }}=10,000 \mathrm{~km} \mathrm{~s}^{-1}$ and takes values of $T=20,000 \mathrm{~K}, w_{\text {wind }}=10 \mathrm{~km} \mathrm{~s}^{-1}$ (which is appropriate for a RSG wind), $t=\left(t_{6 \mathrm{~cm} \text { peak }}-t_{0}\right)$ days from best fits to the radio data for each RSN, and $m=-\delta / 3$ or $m=-(\alpha-\beta-3) / 3$, as appropriate $[31,21]$.

The optical depth term $<\tau_{\text {eff }}^{0.5}>$ used by [25] is extended by [30] and they identify at least three possible absorption regimes: 1) absorption by a homogeneous external medium, 2) absorption by a clumpy or filamentary external medium with a statistically large number of clumps, and 3) absorption by a clumpy or filamentary medium with a statistically small number of clumps. These three cases have different formulations for $\left\langle\tau_{\text {eff }}^{0.5}>\right.$ which are described in detail by [31]. Mass-loss rate estimates from radio absorption obtained in this manner tend to be $\sim 10^{-6} \mathrm{M}_{\odot} \mathrm{yr}^{-1}$ for type $\mathrm{Ib} / \mathrm{c} \mathrm{SNe}$ and $\sim 10^{-4}-10^{-5} \mathrm{M}_{\odot} \mathrm{yr}^{-1}$ for type II SNe. Estimates for some of the best studied $\mathrm{SNe}$ are given in [31]. As discussed in Panagia et al. (2006, preparation) the high mass loss rates measured for SNII confirm that their progenitors are Red Supergiants with original masses above $8 \mathrm{M}_{\odot}$.

\section{Changes in Mass-Loss Rate}

A particularly interesting case of mass-loss from an RSN is SN 1993J, where detailed radio observations are available starting only a few days after explosion (Fig. 3). Van Dyk et al. [24] find evidence for a changing mass-loss rate (Fig. 4) for the presupernova star which was as high as $\sim 10^{-4} \mathrm{M}_{\odot} \mathrm{yr}^{-1}$ approximately 1000 years before explosion and decreased to $\sim 10^{-5} \mathrm{M}_{\odot} \mathrm{yr}^{-1}$ just before explosion, resulting in a relatively flat density profile of $\rho \propto r^{-1.5}$.

Fransson \& Björgsson [6] have suggested that the observed behavior of the $f$-f absorption for SN 1993J could alternatively be explained in terms of a systematic decrease of the electron temperature in the circumstellar material as the SN expands. It is not clear, however, what the physical process is which determines why such a cooling might occur efficiently in SN 1993J, but not in SNe such as SN 1979C and SN 1980K where no such behavior is seen. Also, recent X-ray observations with the ROSAT of SN 1993J indicate a non- $r^{-2}$ CSM density surrounding the SN progenitor [7], with a density gradient of $\rho \propto r^{-1.6}$.

Moreover, changes in presupernova mass-loss rates are not unusual. Montes et al. [10] find that type II SN 1979C had a slow increase in its radio light curve after day $\sim 4300$ (see Fig. 1) and type II SN 1980K showed a steep decline in flux density at 


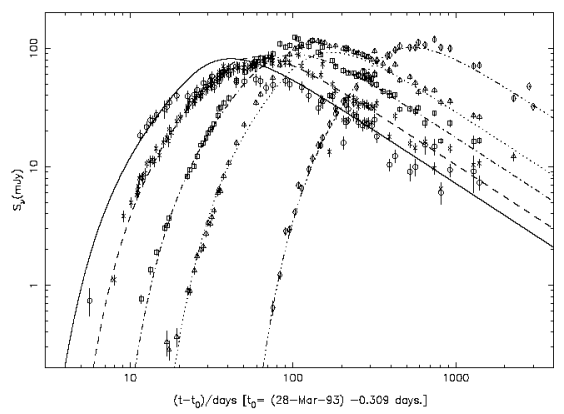

FIGURE 3. Type IIb SN 1993J at $1.3 \mathrm{~cm}(22.5 \mathrm{GHz}$; open circles, solid line $), 2 \mathrm{~cm}(14.9 \mathrm{GHz}$; stars, dashed line), $3.6 \mathrm{~cm}(8.4 \mathrm{GHz}$; open squares, dash-dot line $), 6 \mathrm{~cm}(4.9 \mathrm{GHz}$; open triangles, dotted line), and $20 \mathrm{~cm}(1.5 \mathrm{GHz}$; open diamonds, dash-triple dot line).

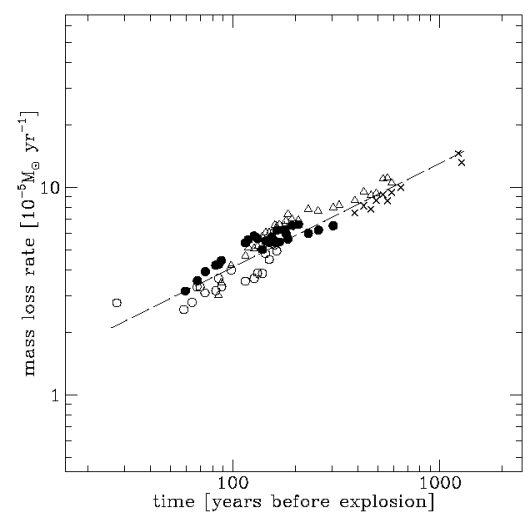

FIGURE 4. Changing mass-loss rate of the presumed red supergiant progenitor to SN 1993J versus time before the explosion.

all wavelengths (see Fig. 2 ) by a factor of $\sim 2$ occurring between day $\sim 3700$ and day $\sim 4900$ [9].

\section{Binary Systems}

In the process of analyzing a full decade of radio measurements from SN 1979C, Weiler et al. [26, 28] found evidence for a significant, quasi-periodic, variation in the amplitude of the radio emission at all wavelengths of $\sim 15 \%$ with a period of 1575 days or $\sim 4.3$ years (see Fig. 1 at age $<4000$ days). They interpreted the variation as due to a minor $(\sim 8 \%)$ density modulation, with a period of $\sim 4000$ years, on the larger, relatively constant presupernova stellar mass-loss rate. Since such a long period is inconsistent with most models for stellar pulsations, they concluded that the modulation 
may be produced by interaction of a binary companion in an eccentric orbit with the stellar wind from the presupernova star.

This concept was strengthened by more detailed calculations for a binary model from [18]. Since that time, the presence of binary companions has been suggested for the progenitors of SN 1987A [14], SN 1993J [15], SN 1994I [12], SN 1998bw [30], and SN $2001 \mathrm{ig}$ [17], indicating that binaries may be common in presupernova systems.

\section{Ionized Hydrogen Along the Line-of-Sight}

A reanalysis of the radio data for SN 1978K from [16] clearly shows flux density evolution characteristic of normal type II SNe. Additionally, the data indicate the need for a time-independent, free-free absorption component. Montes et al. [8] interpreted this constant absorption term as indicative of the presence of HII along the line-of-sight to SN $1978 \mathrm{~K}$, perhaps as part of an HII region or a distant circumstellar shell associated with the SN progenitor. A high-dispersion spectrum of SN $1978 \mathrm{~K}$ at the wavelength range $6530-6610 \AA$ obtained by [4] showed narrow nebular $\mathrm{H} \alpha$ and [N II] emission with a high [N II] $6583 / \mathrm{H} \alpha$ ratio of $0.8-1.3$ indicative of a stellar ejecta nebula. These data suggest that the nebula detected towards SN $1978 \mathrm{~K}$ is probably part of a large, dense, structured circumstellar envelope.

\section{Constraints on Type Ia Supernova Progenitors}

Recently Panagia et al. [13] have discussed the radio observations of 27 Type Ia supernovae (SNIa) observed over two decades with the Very Large Array. No SNIa has been detected so far in the radio, implying a very low density for any possible circumstellar material established by the progenitor, or progenitor system, before explosion. They derive $2 \sigma$ upper limits to a steady mass-loss rate for individual SNIa systems as low as $\sim 3 \times 10^{-8} \mathrm{M}_{\odot} \mathrm{yr}^{-1}$, discriminating strongly against white dwarf accretion via a stellar wind from a massive binary companion in the symbiotic star. However, in the "single degenerate" scenario, a white dwarf accreting from a relatively low mass companion via a high efficiency ( $>60-80 \%$ ) Roche lobe overflow is still consistent with such limits. The "double degenerate" merger scenario also cannot be excluded.

\section{CONCLUSIONS}

The success of the basic parameterization and modeling is shown in the good agreement between the model fits and the data for all subtypes of RSNe. Thus, the radio emission from SNe appears to be relatively well understood in terms of blastwave interaction with a structured CSM and allows description of such progenitor system properties as massloss rate, change in mass-loss rate, filamentation or clumpiness, binarity, and remote HII, as well as estimates of progenitor properties. 


\section{ACKNOWLEDGMENTS}

NP acknowledges support from STScI, through DDRF grants \#82367 and \#82372, and the Observatory of Rome to attend this conference. KWW wishes to thank the Office of Naval Research (ONR) for the 6.1 funding supporting this research.

\section{REFERENCES}

1. Chevalier, R. A. 1982a, ApJ, 259, 302

2. Chevalier, R. A. 1982b, ApJ1, 259, L85

3. Chevalier, R. A. 1998 , ApJ, 499, 810

4. Chu, Y.-H., Caulet, A., Montes, M. J., Panagia, N., Van Dyk, S. D., \& Weiler, K. W. 1999, ApJ1, 512, L51

5. Filippenko, A., Matheson, T., \& Barth, A. 1994, AJ, 108, 222

6. Fransson, C., \& Björgsson, C.-I. 1998, ApJ, 509, 861

7. Immler, S., Aschenbach, B., \& Wang, Q. D. 2001, ApJl, 561, L107

8. Montes, M. J., Weiler, K. W., \& Panagia, N. 1997, ApJ, 488, 792

9. Montes, M. J., Van Dyk, S. D., Weiler, K. W., Sramek, R. A., \& Panagia, N. 1998, ApJ, 506, 874

10. Montes, M. J., Weiler, K. W., Van Dyk, S. D., Sramek, R. A., Panagia, N., \& Park, R. 2000, ApJ, 532,1124

11. Natta, A., \& Panagia, N. 1984, ApJ, 287, 228

12. Nomoto, K., Yamaoka, H., Pols, O.R., van den Heuvel, E., Iwamoto, K., Kumagai, S., Shigeyama, T. 1994, Nature, 371, 227

13. Panagia, N., Van Dyk, S. D., Weiler, K. W., Sramek, R. A., Stockdale, C. J., \& Murata, K. J. 2006, ApJ, 646, 369

14. Podsiadlowski, Ph., Joss, P. C., \& Hsu, J. J. L. 1992, ApJ, 391, 246

15. Podsiadlowski, Ph., Hsu, J., Joss, P., \& Ross, R. 1993, Nature, 364, 509

16. Ryder, S. D., Staveley-Smith, L., Dopita, M., Petre, R., Colbert, E., Malin, D., \& Schlegel, E. 1993, ApJ, 417, 167

17. Ryder, S. D., Sadler, E. M., Subrahmanyan, R., Weiler, K. W., Panagia, N., \& Stockdale, C. J. 2004, MNRAS, 349, 1093

18. Schwarz, D. H., \& Pringle, J. E. 1996, MNRAS, 282, 1018

19. Spyromilio, J. 1994, MNRAS, 266, 61

20. Sramek, R. A., Panagia, N., \& Weiler, K. W. 1984, ApJl, 285, L59

21. Sramek, R. A., \& Weiler, K. W. 2003, Supernovae and Gamma-Ray Bursters, ed. K. Weiler (Berlin: Springer-Verlag LNP 598) p. 145

22. Van Dyk, S. D., Sramek, R. A., Weiler, K. W., \& Panagia, N. 1993a, ApJ, 409, 162

23. Van Dyk, S. D., Sramek, R. A., Weiler, K. W., \& Panagia, N. 1993b, ApJl, 419, L69

24. Van Dyk, S. D., Weiler, K. W., Sramek, R., Rupen, M., \& Panagia, N. 1994, ApJ1, 432, L115

25. Weiler, K., Sramek, R., Panagia, N., van der Hulst, J., \& Salvati, M. 1986, ApJ, 301, 790

26. Weiler, K. W., Panagia, N., \& Sramek, R. A. 1990, ApJ, 364, 611

27. Weiler, K., Van Dyk, S., Panagia, N., Sramek, R., \& Discenna, J. 1991, ApJ, 380, 161

28. Weiler, K., Van Dyk, S., Pringle, J., \& Panagia, N. 1992a, ApJ, 399, 672

29. Weiler, K., Van Dyk, S., Panagia, N., \& Sramek, R. 1992b, ApJ, 398, 248

30. Weiler, K. W., Panagia, N., \& Montes, M. J. 2001, ApJ, 562, 670

31. Weiler, K. W., Panagia, N., Montes, M. J., \& Sramek, R. A. 2002, Ann. Rev. A \& Ap, 40, 387

Williams, C. L., Panagia, N., Lacey, C. K., Weiler, K. W., Sramek, R. A., \& Van Dyk, S. D. 2002 , ApJ, 581, 396 